\title{
Der Mensch schläft nicht fürs Hirn allein
}

Rund ein Drittel seines Lebens verschläft der Mensch. Wer hier Zeitverschwendung wähnt und länger wach zu bleiben sucht, tut sich keinen Gefallen. Denn das Leben wird dadurch nicht verlängert, sondern sehr wahrscheinlich verkürzt.

Laut den Ergebnissen einer Studie, die Forscher der Universität Chicago mit sieben gesunden jungen Erwachsenen unternommen haben, vermindert eine von 8,5 auf 4,5 Stunden reduzierte Schlafdauer die Insulinsensitivität des gesamten Körpers um 16\%. Betrachtet man isoliert die zelluläre Sensitivität der Adipozyten, ist sogar eine Senkung um $30 \%$ zu verzeichnen. Das sind Größenordnungen, die den Unterschied von schlanken zu fettleibigen Menschen bzw. die Differenz von Nichtdiabetikern zu Diabetikern markieren. In der vorliegenden Studie genügte dafür bereits ein über vier Tage hinweg angehäuftes Schlafdefizit.

Die Probanden brachten jeweils vier Tage mit 8,5 Stunden Nachtschlaf und, mit vierwöchigem Abstand, vier Tage mit nur 4,5 Stunden Schlaf zu. Die Schlafdauer wurde polysomnografisch überwacht. Am Ende jeder Phase wurden die Teilnehmer einem Glukosetoleranztest unterzogen. Außerdem entnahmen die Forscher Proben des subkutanen Fettgewebes.

Während der Glukosetoleranztest Auskunft über die allgemeine Insulinsensitivität gab, versuchte man mit den Fettgewebsproben, den molekularen Mechanismen auf die Spur zu kommen, über die das Schlafdefizit die Reaktion der Zellen auf Insulin herabreguliert. Dabei zeigte sich, dass der Mangel an Schlaf die Fähigkeit des Insulins beeinträchtigte, die Spiegel an phosphorylierter Proteinkinase B (Akt) zu erhöhen - ein entscheidender Schritt des Phosphoinositid-3-Kinase-Signalwegs, der die meisten metabolischen Insulinwirkungen vermittelt.

„Vom klinischen Standpunkt aus liegen nun weitere Beweise dafür vor, dass mangelnder Schlaf zur Entwicklung oder Verschlechterung metabolischer Störungen beiträgt", so das Fazit der Autoren.

Broussard JL et al. Ann Intern Med 2012; 157: 549-57

\section{BETAGTE FRAUEN MIT RISIKOFAKTOREN}

\section{Depression wird mit den Jahren schlimmer}

— Fast jede fünfte Frau entwickelt ab dem 65. Lebensjahr innerhalb von 20 Jahren eine Depression.

In einer Studie mit fast 7300 älteren Frauen erkrankte fast jede fünfte Frau über 65 innerhalb von 20 Jahren an einer Depression. Im Vergleich zu Frauen ohne Depression hatten sie zu Beginn der Erkrankung mehr Komorbiditäten und einen ungesunden Lebensstil.

Mithilfe der Geriatrischen Depressionsskala wurden die Teilnehmerinnen psychometrisch beurteilt. Ein Score von mindestens 6 galt als Hinweis auf eine Depression. Je nach Schweregrad der Symptomatik wurden die Frauen vier Gruppen zugeordnet. Die beiden Gruppen mit der schwersten Symptomatik (18,2\% der Frauen) hatten im Verlauf der Studie zunehmend depressive Symptome oder sogar anhaltend schwere Symptome.

Die Wahrscheinlichkeit, im Verlauf von 20 Jahren in einer dieser beiden Gruppen

zu landen, war je nach Einflussfaktor unterschiedlich hoch. In der statistischen Auswertung berücksichtigt wurden Rauchen zu Studienbeginn, Bewegungsmangel, kleines soziales Netz, körperliche Einschränkungen, Herzinfarkt sowie Diabetes und Adipositas.

Das höchste Risiko für eine anhaltend schwere depressive Symptomatik hatten mit einer Odds Ratio (OR) von 16,43 jene Frauen, die körperlich beeinträchtigt waren, gefolgt von Frauen, die rauchten (OR = 7,97), die nur ein kleines soziales Netz (OR $=6,75)$, Diabetes $(O R=3,03)$, Adipositas $(\mathrm{OR}=2,90)$ oder einen Herzinfarkt $(\mathrm{OR}=$

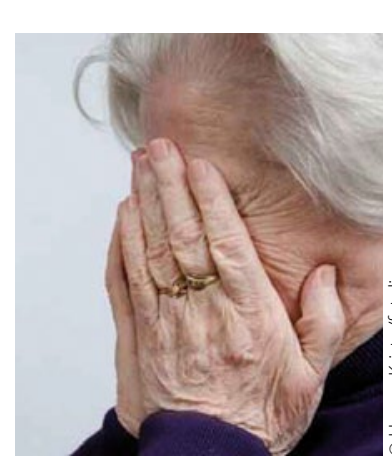

Nach der Menopause steigt das Depressionsrisiko.

\section{2,41 ) hatten.}

Nach Ansicht der Autoren stützen diese Daten die „Vascular-Depression-Hypothese", nach der Gefäßerkrankungen und entsprechende Risikofaktoren die Schwere der Depression sowie die Chronifizierung bis ins hohe Alter begünstigen.

PLE =

Byers AL et al. Arch Gen Psychiatry. 2012; 69(10): 1073-1079

\section{GEWALT DURCH PATIENTEN}

\section{Aggressive Alte?}

— Für viele Beschäftigte in medizinischen Berufen sind Aggressivität und körperliche Gewalt Teil ihres Alltags. Autoren der Universität Hamburg befragten 1973 Beschäftigte deutscher Gesundheits- und Fürsorgedienste, darunter Einrichtungen für Behinderte, Krankenhäuser und geriatrische Pflegeeinrichtungen. 78\% der Befragte gaben an, in den vergangenen zwölf Monaten Verbalattacken der von ihnen Betreuten erlebt zu haben. In Wohnheimen von Menschen mit Behinderungen erlebten die Angestellten diese Form der Aggressivität besonders häufig (86\%). Auch mit physischer Gewalt hatte mehr als jeder Zweite (56\%) Erfahrungen gemacht, 44\% sogar mindestens einmal pro Monat. Die meisten tätlichen Angriffe ereigneten sich in stationären Einrichtungen der Altenpflege (63\%). Dabei hatten jüngere Pflegekräfte ein fast doppelt so hohes Risiko, körperlich attackiert zu werden, wie ältere. Die meisten aggressiven Reaktionen entwickelten sich in einer direkten Pflegesituation, etwa beim Baden, Duschen, bei der Mundhygiene oder beim Anziehen. ST =

Schablon A et al. BMJ Open 2012;2:e001420; doi:10.1136/bmjopen-2012-001420 